\title{
Effect of floating axial holder on oscillating cold forging of spline shaft
}

\author{
N Y Ben ${ }^{1,2}$, Q Zhang ${ }^{1}$ and M G Lee ${ }^{2}$ \\ 1 School of Mechanical Engineering, Xi'an Jiaotong University, Xi'an 710049, China \\ 2 Department of Materials Science and Engineering, Seoul National University, 1 Gwanak-ro, Gwanak-gu, Seoul 08826, Republic of Korea
}

\begin{abstract}
Oscillating technique is applied into the axial forging process of spline shaft to decrease the forming load and improve the quality of products. A floating axial holder with dwell force is designed to control the material flow. The influences of the floating axial holder have been analysed by finite element (FE) simulation and then verified by experiments. A FE model that considers material property change, elastic recovery and elastoplastic friction was built. The constitutive material model, which is mainly composed of variable elastic modulus and rate-dependent hardening, was used to simulate changes in material properties. Results showed that material flow was improved by decreasing dwell force. Tooth shape can be controlled by changing the dwell force of floating axial holder. The nonlinear function between addendum circle diameter and dwell force has been found. Hence, an optimal value of dwell force was determined considering the forming force, forming defects and tooth shape. Based on the simulating results, the optimal and irrational parameters of dwell force and frequency have been compared in the experiments. The typical defects of flash and accumulation can be eliminated by using the floating axial holder with optimal parameters.
\end{abstract}

\section{Introduction}

Plastic forming methods such as rolling, spinning and extrusion are superior to machining method in spline shaft manufacturing owing to the higher strength of products [1-3]. However, spline modulus is limited in rolling and spinning because severe deformation is hard to achieve in such radial rotary forming $[4,5]$. In extrusion process, the material is forced to shaped parts through the dies which are exposed to high loads. Spline modulus is directly determined by the shape of the die. But the billet will be bent easily during extrusion because of the high friction [6].

To decrease the forming load and improve the quality of products, oscillating technique with low frequency (5$20 \mathrm{~Hz}$ ) is applied in the forming process. Groche and $\mathrm{He} \beta$ examined the method to control the final geometry of the product in oscillating cold forging process [7]. Numerous studies have been done to find the reasons of force reduction in the oscillating forming process. Improvement of friction condition and softening behaviour of material properties are two main reasons [810]. Ben et al. investigated the real contact area in oscillating bulk forming process by corrosion method [11]. The re-lubrication during the back step was recorded by the different degrees of corrosion.

Extrusion process needs to be changed to match the application of oscillating technology. Contrary to conventional extrusion, the billet is steady but the die feeds with a ram motion in oscillating forging as shown in figure 1. Because the die goes in the opposite direction in the back step, reverse friction will cause the material to

flow back. Hence, an axial holder is necessary to control the material flow. The effects of the axial holder are similar to the blank holder in deep drawing process which is usually used to guarantee stability and control friction force [12]. Furthermore, the axial holder should cooperate with the cyclic reversal of force [13].

In this paper, a floating axial holder with dwell force is designed to control the material flow in oscillating cold forging of spline shaft. The influences of the floating axial holder were analysed by finite element (FE) simulation and then verified by experiments. A FE model that considers material property change, elastic recovery, and elastoplastic friction was built. The constitutive material model, which is mainly composed of variable elastic modulus and rate-dependent hardening models, was used to simulate changes in material properties. The effects of dwell force on forming force, material flow and tooth shape were studied. Based on the results of simulation, the optimal value of dwell force have been found and then verified in the experiments.

$$
\text { (a) Extrusion }
$$

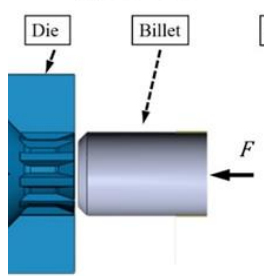

Figure 1. Diagram of extrusion process (a) and oscillating forging process $(b)$ of spline shaft. 


\section{FE model}

\subsection{Constitutive model}

Given that the forming die reciprocates in the oscillating forming process, the material is under a cyclic loading-unloading-loading (LUL) condition with a changing strain rate. Therefore, two important effects, namely, the changing of Young's modulus and strain rate sensitivity, should be considered in the material constitutive model. Young's modulus decreases with increasing number of unloading. The change of elastic modulus can be defined by a function of the equivalent plastic strain, also known as the chord model. Yoshida et al. [14] proposed a model to describe the changing Young's modulus related with the equivalent strain, as shown in equation (1):

$$
E_{a v}=E_{0}-\left(E_{0}-E_{a}\right)\left[1-\exp \left(-\xi \varepsilon_{0}^{P}\right)\right]
$$

where $E_{0}$ is the initial Young' $\mathrm{s}$ modulus in elastic region, $E_{\mathrm{a}}$ is the saturation Young' $\mathrm{s}$ modulus, $\varepsilon_{0}$ is the equivalent plastic strain and $\xi$ is the material constant. During bulk forming, the material can be regarded as isotropic. Thus, the convenient Mises criteria is sufficient as the yield criteria. Considering the effect of strain rate, one of the most widely used rate-dependent hardening models, 'Johnson-Cook' (JC) model, is used to describe the mechanical behaviour of alloys as shown in equation (2):

$$
\sigma_{0}=\left(A+B \varepsilon^{n}\right)\left[1+C \ln \left(\frac{\dot{\varepsilon}}{\dot{\varepsilon}_{0}}\right)\right]
$$

where $\sigma_{0}$ is the yield stress. The equivalent strain rate $\dot{\varepsilon}$ and the equivalent strain $\varepsilon$ are read from the results of the flow law. $A, B, C$ and $n$ are material constants.

The parameters in the constitutive model can be determined using a uniaxial material test. The material is AISI 1045A which is the same with that in the experiments. Three different strain rates of 0.01, 0.001 and $0.0001 \mathrm{~s}-1$ were applied to the monotonic tests. During the LUL test, the frequency of each unloading was $1 \%$ strain. Hence, the parameters in the constitutive model was determined by the true stressstrain curves as shown in Table 1.

\subsection{FE model}

The FE model was built by forging simulating software FORGE while the constitutive model and friction model can be realized by subroutine in the function of secondary development. The geometry parameters of the spline are shown in Table 2. Given their symmetry, tenth of the die and billet were developed as the 3D FE model, as shown in figure 3. The rigid die moved with an oscillating way. Three frequency values, $10 \mathrm{~Hz}, 15 \mathrm{~Hz}$ and $20 \mathrm{~Hz}$ were utilized on this die. The velocity of the die was $18 \mathrm{~mm} / \mathrm{s}$. Thus, the frequency corresponded to the amplitude of 1.2-0.6 $\mathrm{mm}, 0.9-0.45 \mathrm{~mm}$ and $0.6-0.3 \mathrm{~mm}$ in the formingbacking cycle, respectively. The bottom of the billet was fixed by the manipulator during the process. The element type of billet was tetrahedral mesh of which the size is $0.3 \mathrm{~mm}$ in forming region $\mathrm{mm}$ and $0.8 \mathrm{~mm}$ in other region. To analyse the effects of axial holding force and find the optimal parameters, four different values of dwell force, $0.1,0.3,0.5$ and 0.7 tones, were applied on the floating axial holder.

The friction condition is related to the elastoplastic yield criterion and sliding velocity [15]. Therefore, the IFUM model was chosen to describe the friction shear stress between contact pairs during plastic deformation. The IFUM model can distinguish elastic deformation from plastic deformation. Besides, the relative relocity between the interfaces is considered. The mathematical formula of the friction model is given by the following equation (3):

$$
\tau=\left[0.3\left(1-\frac{\sigma_{E q}}{\sigma_{0}}\right) \cdot \sigma_{N}+\sqrt{3} \cdot m \cdot \frac{\sigma_{E q}}{\sigma_{0}} \cdot\left(1-\exp \frac{-\left|\sigma_{N}\right|}{\sigma_{0}}\right)\right] \exp \frac{-1}{2}\left(\frac{v_{r e l}}{5}\right)^{2}
$$

where $\tau$ is the friction force, $m$ is the friction factor, $\sigma_{N}$ is the normal stress, $\sigma_{E q}$ is the equivalent stress, $\sigma_{o}$ is the yield stress and $v_{r e l}$ is the relative sliding velocity. Except the friction factor $m$, others are got from the simulation software FORGE. The friction factors have been determined by previous research [18], which are $0.15,0.125,0.1$ and 0.08 corresponding to the frequencies are $0 \mathrm{~Hz}, 10 \mathrm{~Hz}, 15 \mathrm{~Hz}$ and $20 \mathrm{~Hz}$, respectively.

Table 1. Parameters in the constitutive model.

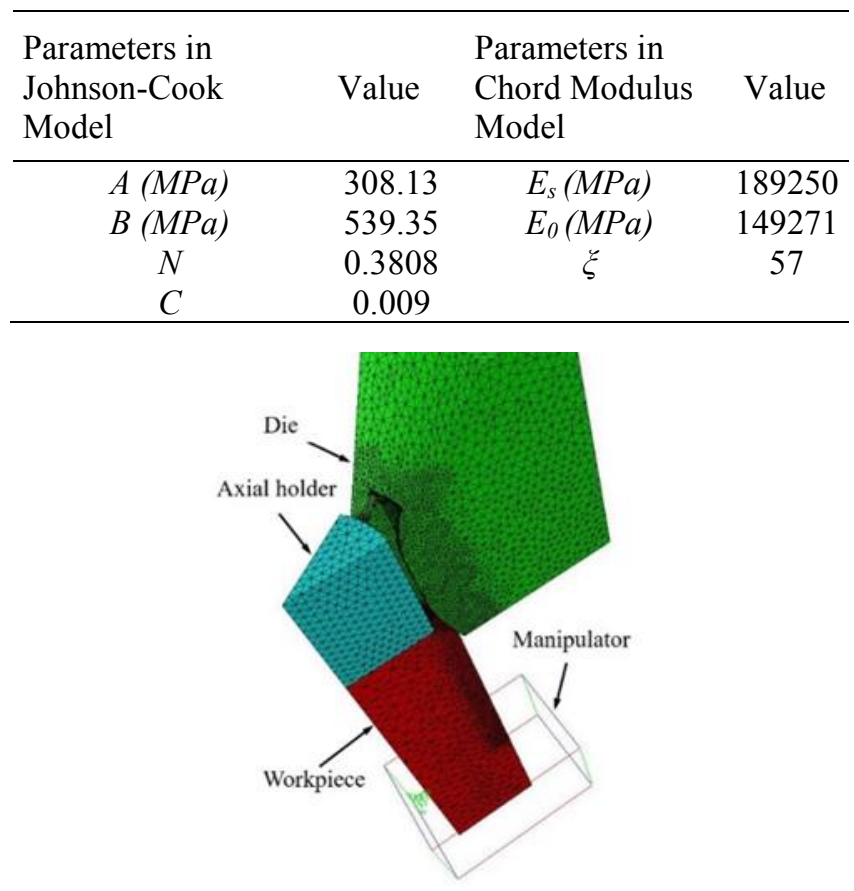


Figure 2. The FE model of oscillating cold forging of spline shaft.

Table 2. Parameters of the spline.

\begin{tabular}{lc}
\hline \multicolumn{1}{c}{ Parameters in } \\
Johnson-Cook Model & Value \\
\hline Tooth number, $z$ & 10 \\
Normal module, $m_{n}$ & 1.75 \\
Pressure angle at reference circle, $\alpha_{n} /{ }^{\circ}$ & 30 \\
Diameter of addendum circle, $D_{a} / \mathrm{mm}$ & 17.90 \\
Diameter of dedendum circle,$D_{f} / \mathrm{mm}$ & 15.75 \\
\hline
\end{tabular}

\section{Results and discussion}

\subsection{Material flow}

Material flow is a key factor for the quality of products. The mechanism of material flow provides the basis for modifying process parameters. Take the process with the frequency of $10 \mathrm{~Hz}$ and the dwell force of $0.7 \mathrm{~T}$ for example, the Von Mises stress and velocity vector distribution during the whole process is shown in figure 3. In the feeding stroke, the material flow can be divided by three parts (figure 3 (a)), which are flowing to the tooth tip, accumulating with the die and against the forming direction. The last one can be controlled by the axial holder directly. As is shown in figure 3 (b), the elastic recovery happened in the backing stroke which would be against by the axial holder. The elastic recovery played an important role in the oscillating forming process. In the backing stroke, lubricant refilled into the gap which are related to the elastic recovery. The products formed by the same frequency but different dwell forces were shown in figure 4 . The dwell force prevents the material flowing against the forming direction. However, if the dwell force was too large, the material would accumulate to the bottom. The defects of flash and accumulation can be found in the final product as shown in figure 4 (c). Besides, material flow was also influenced by the oscillating frequency. The products formed by the different frequencies were shown in figure 5 when the dwell force was $0.5 \mathrm{~T}$. Material flow were improved by increasing frequency. The defects of accumulation and flash were eliminated in the process with the frequency of $20 \mathrm{~Hz}$.

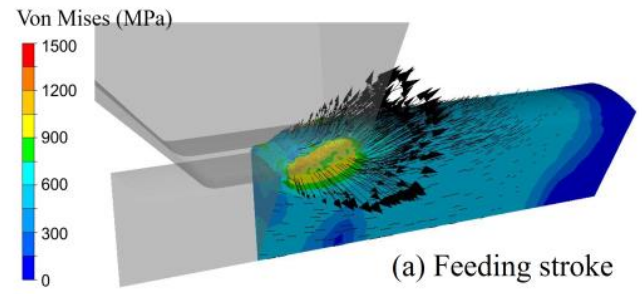

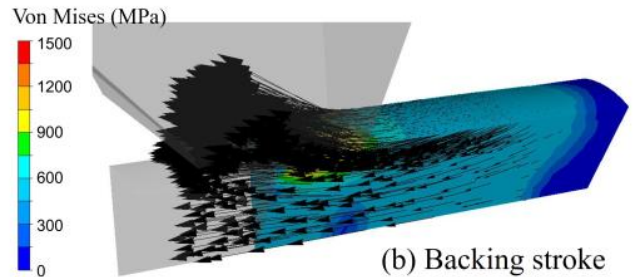

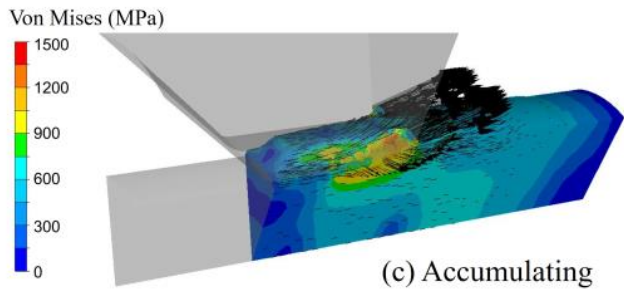

Figure 3. Velocity vectors and distribution of Von Mises stress in the steps of feeding (a), backing (b) and accumulating (c) when the frequency was $10 \mathrm{~Hz}$ and dwell force was $0.7 \mathrm{~T}$.
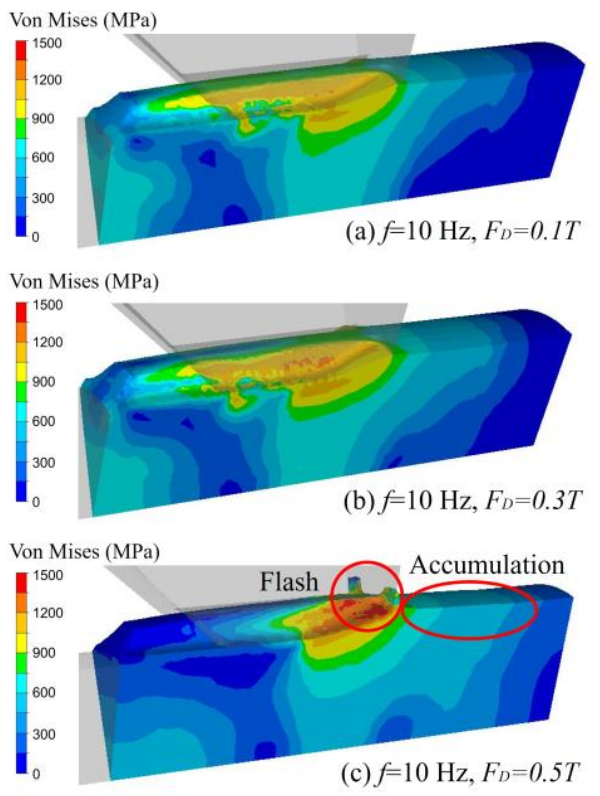

Figure 4. Distribution of Von Mises stress of the final products formed by the frequency of $10 \mathrm{~Hz}$ when the dwell force was $0.1 \mathrm{~T}(\mathrm{a}), 0.3 \mathrm{~T}(\mathrm{~b})$ and $0.5 \mathrm{~T}$ (c).

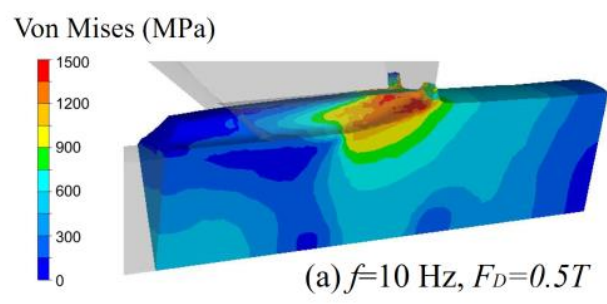

Von Mises (MPa)

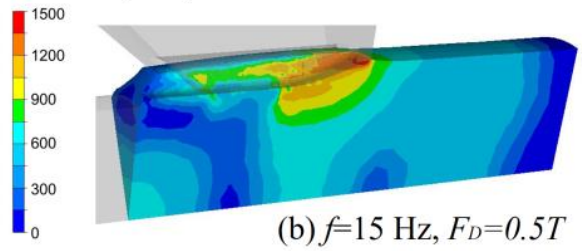




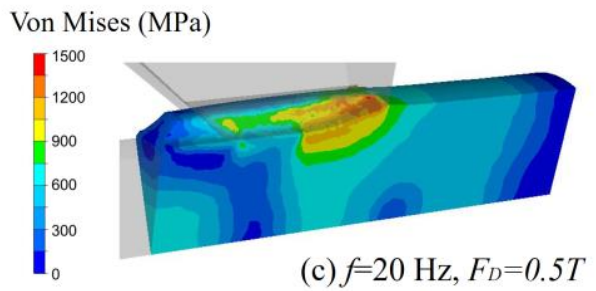

Figure 5. Distribution of Von Mises stress of the final products formed by the dwell force of $0.5 \mathrm{~T}$ when the frequency was $10 \mathrm{~Hz}$ (a), $15 \mathrm{~Hz}$ (b) and $20 \mathrm{~Hz}$ (c).

\subsection{Tooth shape}

The advantages of the axial holder are reflected in the control of the tooth shape. The analysis above indicates that the axial holder controls the tooth shape by controlling the material flow. Suppose there was no axial holder, the top of the shaft would be free. Then the material would flow against the forming direction due to the smallest resistance, i.e., the material would be hard to flow to the tooth tip. However, the tooth shape is the fundamental target, especially the addendum circle diameter, $D_{a}$. The average $D_{a}$ of the spline shafts of which the requirement was $17.9 \mathrm{~mm}$ were shown in figure 6 . The $D_{a}$ increases with the increasing dwell force and frequency. The result can be nonlinearly fitted to a formula as shown in equation (4). According to the analysis before, the frequency of 20 $\mathrm{Hz}$ and a lower dwell force is better to the material flow. Therefore, dwell force gets its optimal value of 0.401 when the $D_{a}$ reach the standard.

$D_{a}=17.426+0.57 \ln \left(3.486 F_{D}+0.9\right)$

\section{Experimental verification}

A horizontal hydraulic oscillating extrusion machine with a floating axial holder was designed for the axial forging of spline shaft. The oscillating forging process was performed by the hydraulic servo toroidal cylinder, which allows the floating axial holder to pass through. The dwell force of the axial holder is supplied by the pressure maintaining cylinder. The measurement system was composed of force sensor, grating-rule sensor, and computer. At the beginning, the workpiece was clamped by a clamping device. Then, the floating axial holder pushed against the workpiece. During the process, the die was moved ahead and back by the servo cylinder. The type of lubricant is liquid lubricant, the parameter $\mu$ of which is $0.485 \mathrm{~Pa} \cdot \mathrm{S}$.

Based on the analysis of the simulations, the optimal parameters of which frequency is $20 \mathrm{~Hz}$ and dwell force is $0.4 \mathrm{~T}$ are applied to the experiments. To compare the differences, the contrast experiment has been done with irrational parameters of which frequency is $10 \mathrm{~Hz}$ and dwell force is $0.7 \mathrm{~T}$. As is shown in figure 7 , the product formed by optimal parameters (figure 7 (a)) shows a better performance quality than that formed by irrational parameters (figure 7 (b)). The average addendum circle diameter of the spline made by optimal parameters was $17.912 \mathrm{~mm}$ which met the standard. The typical defect of flash was found in the experiments with irrational parameters as shown in figure 7 (c). Besides, the workpiece was bent due to the large forming force caused by irrational parameters.

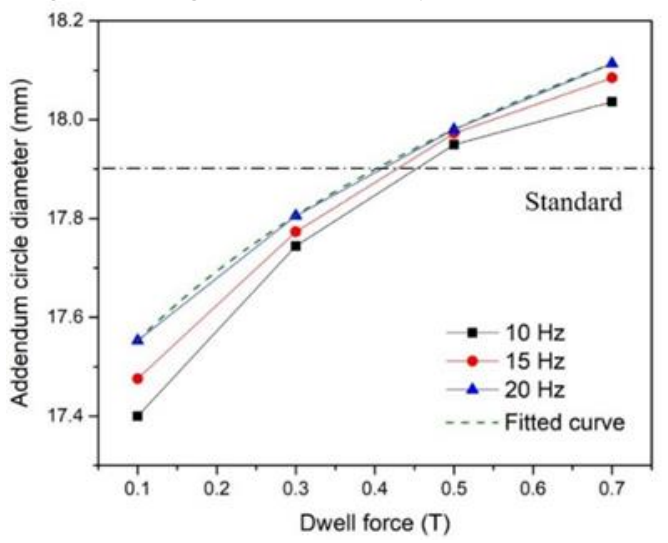

Figure 6. Average addendum circle diameter of products formed by different parameters.

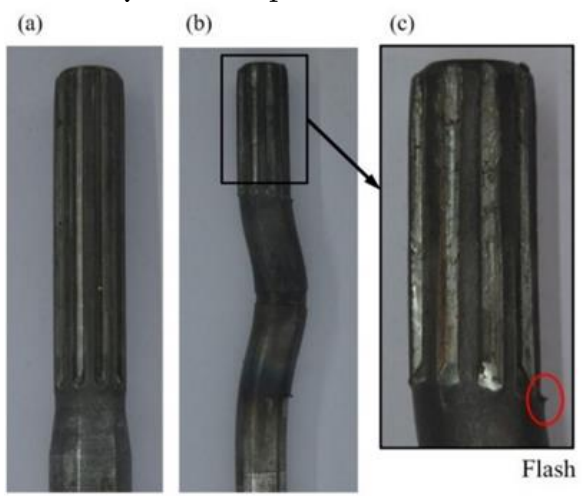

Figure 7. The spline shaft formed by the optimal parameters (a) and the worst parameters (b). Flash and scratches were found in the irrational parameters (c).

\section{Conclusions}

The floating axial holder with a dwell force was designed to control the material flow for tooth shaping in oscillating axial forging process of spline shaft. The optimal process parameters were analysed by simulation. Experiments show that the defects of flash and accumulation can be eliminated by using the floating axial holder with optimal parameters.

\section{References}

1. Zhuang X, Sun X, Xiang H, Xia M, Zhao Z 2016 Int. J. Adv. Manuf. Tech. 84 2331-45

2. Zhang Q, Mu D, Jin K, Lu Y 2014 J. Mater. Process. Tech. 214 2971-84 
3. Wang W, Zhao J, Zhai R, Ma R 2016 Int. J. Adv. Manuf. Tech. 88 3163-73

4. Cui M C, Zhao S D, Chen C, Zhang D W 2017 Int. J. Adv. Manuf. Tech. 90 3001-11

5. Zhang Q, Zhang C, Zhang M J, Zhu C C 2015 Int. J. Adv. Manuf. Tech. 81 1601-18

6. Yuan A 2009 Int. J. Adv. Manuf. Tech. 41 4617

7. Groche P, Heß B, 2014 CIRP Ann-Manuf. Techn. 63 285-8

8. Siegert K, Möck A 1996 J. Mater. Process. Tech. 60 657-60

9. Matsumoto R, Sawa S, Utsunomiya H, Osakada K 2011 CIRP Ann-Manuf. Techn. 60 315-8
10. Siegert K, Ulmer J 2001 J. Eng. Mater-T. ASME 123 517-23

11. Ben N Y, Zhang Q, Meng D A, Lee M G 2018 J. Mater. Process. Tech. 253 178-94

12. Sheng Z Q, Jirathearanat S, Altan T 2004 Int. J. Mach. Tool. Manu. 44 487-94

13. Ali S, Hinduja S, Atkinson J, Bolt P, Werkhoven R 2008 Int. J. Mach. Tool. Manu. 48 558-64

14. Yoshida F, Uemori T, Fujiwara K 2002 Int. J. Plasticity $18633-59$

15. Zhang Q, Ben, N Y, Yang K 2017 J. Mater. Process. $\quad$ Tech. $244 \quad 166-77$ 